\title{
Towards gauge theories in four dimensions
}

\author{
Roger J. Hernández-Pinto, ${ }^{a}$ Germán F.R. Sborlini ${ }^{a, b}$ and Germán Rodrigo ${ }^{a}$ \\ ${ }^{a}$ Instituto de Física Corpuscular, \\ Universitat de València - Consejo Superior de Investigaciones Científicas, \\ Parc Cientific, E-46980 Paterna, Valencia, Spain \\ ${ }^{b}$ Departamento de Física and IFIBA, FCEyN, Universidad de Buenos Aires, \\ Pabellón 1 Ciudad Universitaria, 1428, Capital Federal, Argentina \\ E-mail: rogerjose.hernandez@ific.uv.es, german.sborlini@ific.uv.es, \\ german.rodrigo@csic.es
}

ABSTRACT: The abundance of infrared singularities in gauge theories due to unresolved emission of massless particles (soft and collinear) represents the main difficulty in perturbative calculations. They are typically regularized in dimensional regularization, and their subtraction is usually achieved independently for virtual and real corrections. In this paper, we introduce a new method based on the loop-tree duality (LTD) theorem to accomplish the summation over degenerate infrared states directly at the integrand level such that the cancellation of the infrared divergences is achieved simultaneously, and apply it to reference examples as a proof of concept. Ultraviolet divergences, which are the consequence of the point-like nature of the theory, are also reinterpreted physically in this framework. The proposed method opens the intriguing possibility of carrying out purely four-dimensional implementations of higher-order perturbative calculations at next-to-leading order (NLO) and beyond free of soft and final-state collinear subtractions.

KEYwORDS: NLO Computations

ARXIV EPRINT: 1506.04617

Open Access, (C) The Authors.

Article funded by $\mathrm{SCOAP}^{3}$. 


\section{Contents}

1 Introduction 1

2 Review of the loop-tree duality 2

3 Dual cancellations 3

4 Real-Virtual mapping and cancellation of infrared singularities $\quad 6$

$\begin{array}{lll}5 & \text { Ultraviolet renormalization } & 9\end{array}$

6 Conclusions 10

\section{Introduction}

The most important Quantum Field Theories (QFT) for describing elementary particle physics are gauge theories. Quantum Yang-Mills theories [1] were introduced about sixty years ago and are now the foundation of most of elementary particle theory. One of the main difficulties to extract theoretical predictions from perturbative calculations at higher orders is the spread of infinities. In the high-energy regime, there are ultraviolet (UV) divergences related to the point-like nature of the theory. Due to the preservation of gauge invariance, dimensional regularization (DREG) [2-5], that consists in changing the number of space-time dimensions from 4 to $d=4-2 \epsilon$, is the customary regularization method to overcome this problem. The UV singularities in four dimensions manifest as poles in the dimensional parameter $\epsilon$, and are easily removed by renormalization. The real challenge is, however, in the infrared (IR) regime. Unresolved radiation of massless particles (soft and collinear) is physically degenerate with the absence of emission, while from the theory point of view they are described as different final states. This leads to further infinities that are also regularized in DREG.

The standard approach [6-14] to remove the IR singularities consists of adding to the real radiation contribution suitable subtraction terms that mimic the IR behavior of the emission scattering amplitudes and render them finite in the IR limit. The same subtraction quantities, integrated over the phase-space of the extra radiation, are subtracted back from the virtual corrections. The sum of both contributions leads to finite theoretical predictions for physical observables, if all the degenerate states are considered in the sum. In this paper, we describe an alternative approach which is based on the loop-tree duality (LTD) theorem [15-22]. We propose a new method that combines simultaneously virtual and real corrections, following in fact the original aim of the LTD method. We present here the first practical realization of LTD that we illustrate with some reference examples. This fact has also a strong implication: the possibility of carrying out purely four-dimensional 
implementations free of soft and final-state collinear subtractions. Unlike the method proposed in ref. [23], LTD does not require any smearing in the total energy to combine real and virtual corrections. Likewise, massless internal propagators are kept massless in LTD, as opposed to the four dimensional implementations presented in refs. [24-26].

\section{Review of the loop-tree duality}

The LTD theorem [15] establishes a direct dual relation between one-loop $N$-leg scalar integrals or scattering amplitudes in any relativistic, local and unitary QFT with corresponding tree-level objects integrated in a modified phase-space that resemble real corrections. Specifically, the dual representation of a $N$-leg scalar one-loop integral consists of the sum of $N$ dual integrals:

$$
L^{(1)}\left(p_{1}, \ldots, p_{N}\right)=-\sum_{i \in \alpha_{1}} \int_{\ell} \tilde{\delta}\left(q_{i}\right) \prod_{j \in \alpha_{1}, j \neq i} G_{D}\left(q_{i} ; q_{j}\right),
$$

where $G_{D}\left(q_{i} ; q_{j}\right)=\left(q_{j}^{2}-m_{j}^{2}-i 0 \eta \cdot k_{j i}\right)^{-1}$ are dual propagators, with $i, j \in \alpha_{1}=\{1,2, \ldots N\}$. The momenta of the internal lines are denoted $q_{i, \mu}=\left(q_{i, 0}, \mathbf{q}_{i}\right)$, where $q_{i, 0}$ is the energy (time component) and $\mathbf{q}_{i}$ are the spacial components. They are defined as $q_{i}=\ell+k_{i}$ with $\ell$ the loop momentum and $k_{i}=p_{1}+\ldots+p_{i}$. The four-momenta of the external legs are $p_{i}$, which are taken as outgoing, with $k_{N}=0$ by momentum conservation. The loop integration measure reads

$$
\int_{\ell}^{\bullet}=-i \mu^{4-d} \int \frac{d^{d} \ell}{(2 \pi)^{d}} \bullet
$$

The delta function $\tilde{\delta}\left(q_{i}\right) \equiv 2 \pi i \theta\left(q_{i, 0}\right) \delta\left(q_{i}^{2}-m_{i}^{2}\right)$ sets the internal lines on-shell and is the result of selecting the poles of the Feynman propagators with positive energy $q_{i, 0}$ and negative imaginary part by applying the Cauchy residue theorem along a contour that is closed in the lower half of the complex plane. LTD is equivalent to perform the loop integration in the $d-1$ dimensional space defined by the forward on-shell hyperboloids, $G_{F}\left(q_{i}\right)^{-1}=\left(q_{i}^{2}-m_{i}^{2}+i 0\right)=0$ and $q_{i, 0}>0$, or forward light-cones for massless propagators.

The key feature of the dual representation in eq. (2.1) is that the usual Feynman propagators have been replaced by dual propagators whose $+i 0$ prescription depends now on the sign of $\eta \cdot k_{j i}$, where $\eta$ is a future-like vector, $\eta^{2} \geq 0$, with positive definite energy $\eta_{0}>0$, and $k_{j i}=q_{j}-q_{i}$. The idea of having different $+i 0$ prescriptions for different propagators is at first sight astonishing, but indeed it is a necessary condition for the consistency of the method. As shown in ref. [18], the integrand in eq. (2.1) becomes singular at the intersection of forward on-shell hyperboloids (light-cones for massless propagators), and forward with backward $\left(q_{j, 0}<0\right)$ on-shell hyperboloids. Those singularities lead to integrable threshold singularities or non-integrable soft and collinear divergences. In the forwardforward case, there is a cancellation of singularities among different dual contributions, and the change of sign of the dual prescription plays a central role in that cancellation. In the forward-backward case, the singularities remain constrained to a compact region of the loop three-momentum and admit a nice physical interpretation in terms of causality, which is indeed consistent with the Cutkosky rule. These singularities occur only in one 

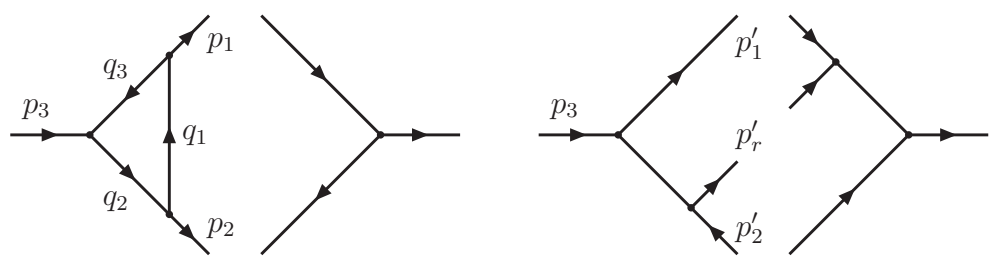

Figure 1. Kinematic configuration of the three-point function for the process $p_{3} \rightarrow p_{1}+p_{2}$, its interference with the Born amplitude (left), and interference of real radiation contributions with $p_{3} \rightarrow p_{1}^{\prime}+p_{2}^{\prime}+p_{r}^{\prime}$ (right).

direction, the direction of the chosen internal momentum flow, when the on-shell virtual particle is emitted and interacts with another outgoing virtual on-shell particle $\left(q_{j, 0}<0\right)$ after loosing energy by radiating external particles.

\section{Dual cancellations}

To validate the LTD method with an explicit example, we focus our attention to the simplest case of the scalar three-point function, with $p_{3} \rightarrow p_{1}+p_{2}$ as external momenta and $p_{3}^{2}=s_{12}>0 ; p_{1}, p_{2}$ and the internal momenta are taken massless. The loop integral is defined by the internal momenta $q_{1}=\ell+p_{1}, q_{2}=\ell+p_{12}$ and $q_{3}=\ell$, according to figure 1. We proceed step by step, by integrating first in DREG and then by repeating the calculation in a suitable representation where the limit $\epsilon \rightarrow 0$ can safely be taken at the integrand level. The dual representation of the scalar three-point function consists of three contributions:

$$
I_{i}=-\int_{\ell} \tilde{\delta}\left(q_{i}\right) \prod_{j \neq i} G_{D}\left(q_{i} ; q_{j}\right), \quad i, j=1,2,3 .
$$

Assuming $p_{1}$ oriented along the positive $z$-axis, $p_{2}$ in the opposite direction, the on-shell loop momenta are parametrized as

$$
q_{i}^{\mu}=\frac{\sqrt{s_{12}}}{2} \xi_{i, 0}\left(1,2 \sqrt{v_{i}\left(1-v_{i}\right)} \mathbf{e}_{i, \perp}, 1-2 v_{i}\right),
$$

with $\xi_{i, 0} \in[0, \infty), v_{i} \in[0,1]$, and $\mathbf{e}_{i, \perp}$ a unit vector in the transverse space. Taking into account these parametrizations the scalar products are given by $2 q_{i} \cdot p_{1} / s_{12}=\xi_{i, 0} v_{i}$, and $2 q_{i} \cdot p_{2} / s_{12}=\xi_{i, 0}\left(1-v_{i}\right)$, and the dual integrals are written as

$$
\begin{aligned}
& I_{1}=\frac{1}{s_{12}} \int d\left[\xi_{1,0}\right] d\left[v_{1}\right] \xi_{1,0}^{-1}\left(v_{1}\left(1-v_{1}\right)\right)^{-1}, \\
& I_{2}=\frac{1}{s_{12}} \int d\left[\xi_{2,0}\right] d\left[v_{2}\right] \frac{\left(1-v_{2}\right)^{-1}}{1-\xi_{2,0}+i 0}, \\
& I_{3}=-\frac{1}{s_{12}} \int d\left[\xi_{3,0}\right] d\left[v_{3}\right] \frac{v_{3}^{-1}}{1+\xi_{3,0}},
\end{aligned}
$$

where we defined the $d$-dimensional integration measures as

$$
\begin{aligned}
d\left[\xi_{i, 0}\right] & =\frac{\mu^{2 \epsilon}(4 \pi)^{\epsilon-2}}{\Gamma(1-\epsilon)} s_{12}^{-\epsilon} \xi_{i, 0}^{-2 \epsilon} d \xi_{i, 0}, \\
d\left[v_{i}\right] & =\left(v_{i}\left(1-v_{i}\right)\right)^{-\epsilon} d v_{i}
\end{aligned}
$$



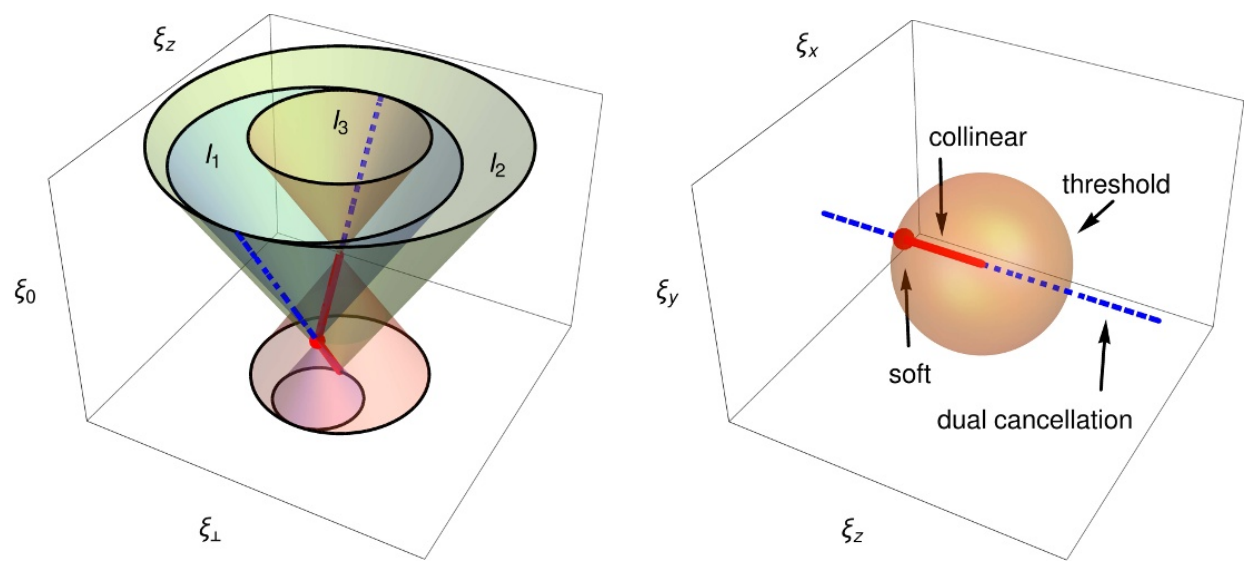

Figure 2. Light-cones of the massless three-point function in the loop coordinates $\ell=$ $\sqrt{s_{12}} / 2\left(\xi_{0}, \xi_{\perp}, \xi_{z}\right)$, with $\xi_{\perp}=\sqrt{\xi_{x}^{2}+\xi_{y}^{2}}$ (left). Each forward light-cone correspond to the integration region of one dual integral $I_{i}$, and the intersection with the other light-cones generates the singularities of the integrand. Location of threshold and IR singularities in the loop threemomentum space (right).

and the dual vector was taken as $\eta^{\mu}=(1, \mathbf{0})$. Figure 2 (left) shows a graphical representation of the corresponding light-cones and their intersections where threshold and IR singularities appear. In eq. (3.3), only the dual prescription of the dual integral $I_{2}$ has been kept explicitly, as it regulates a threshold singularity. The dual prescription of the other propagators is required for the consistent partial cancellation of singularities among dual integrals [18], but can be removed for this practical calculation.

The explicit computation in DREG of the total integrals in eq. (3.3) gives

$$
I_{1}=0, \quad I_{2}=\widetilde{c}_{\Gamma} \frac{\mu^{2 \epsilon}}{2 \epsilon^{2}} s_{12}^{-1-\epsilon} e^{i 2 \pi \epsilon}, \quad I_{3}=\widetilde{c}_{\Gamma} \frac{\mu^{2 \epsilon}}{2 \epsilon^{2}} s_{12}^{-1-\epsilon},
$$

with

$$
\widetilde{c}_{\Gamma}=c_{\Gamma} \frac{1}{\cos (\pi \epsilon)}, \quad c_{\Gamma}=\frac{\Gamma(1+\epsilon) \Gamma^{2}(1-\epsilon)}{(4 \pi)^{2-\epsilon} \Gamma(1-2 \epsilon)},
$$

where $\widetilde{c}_{\Gamma}$ is the volume factor of the phase-space integrals, and $c_{\Gamma}$ is the volume factor of the loop integrals. The dual integral $I_{1}$ vanishes because it is scaleless, and following the usual convention in DREG it is analytically continued by matching its UV and IR poles, i.e. $\epsilon_{\mathrm{UV}}=\epsilon_{\mathrm{IR}}$. Summing up the results in eq. (3.5) we obviously obtain the well-known result

$$
L^{(1)}\left(p_{1}, p_{2},-p_{3}\right)=\int_{\ell} \prod_{i=1}^{3} G_{F}\left(q_{i}\right)=-c_{\Gamma} \frac{\mu^{2 \epsilon}}{\epsilon^{2}}\left(-s_{12}-i 0\right)^{-1-\epsilon} .
$$

At this point, we start the discussion by isolating the singular regions of the loop integrand, which are bounded to a compact region of the loop three-momentum, as it can be seen from figure 2 (right). The dual integral $I_{1}$ develops a soft singularity at $\xi_{1,0}=0$ and a collinear singularity at $v_{1}=0$ with $\xi_{1,0} \in[0,1]$, namely $\xi_{z} \in[-1,0]$; the collinear singularity at $\xi_{z}>0$ cancels with the other dual contributions. Likewise, the dual integral $I_{2}$ is collinear at $v_{2}=1$ with $\xi_{2,0} \in[0,1]$, or $\xi_{z} \in[-1,0]$, and undergo cancelling collinear divergences at $\xi_{z}<-1$. The integrand of $I_{2}$ gets in addition a threshold singularity at $\xi_{2,0}=1$, which prevents us from having a well defined integral if we restrict the integration 
region by $\xi_{2,0}=1$. Consequently, we introduce an energy-cut $w$, with $w$ close to zero, and define the following soft and collinear contributions to the dual integrals

$$
\begin{aligned}
& I_{1}^{(\mathrm{s})}=I_{1}\left(\xi_{1,0} \leq w\right), \\
& I_{1}^{(\mathrm{c})}=I_{1}\left(w \leq \xi_{1,0} \leq 1 ; v_{1} \leq 1 / 2\right), \\
& I_{2}^{(\mathrm{c})}=I_{2}\left(\xi_{2,0} \leq 1+w ; v_{2} \geq 1 / 2\right) .
\end{aligned}
$$

The soft integral $I_{1}^{(\mathrm{s})}$ contains the soft singularity of the dual integral $I_{1}$, and its collinear singularities close to the soft region, $\xi_{1,0}<w$. Once the soft part is removed, the remaining collinear singularities of $I_{1}$ are contained in the collinear integral $I_{1}^{(\mathrm{c})}$. It is necessary to extend the integration limits of the collinear integral $I_{2}^{(\mathrm{c})}$ beyond the strict collinear singular region of $I_{2}$ in order to treat properly its threshold singularity at $\xi_{2,0}=1$. Integrating these contributions in DREG, we obtain the following results:

$$
\begin{aligned}
& I_{1}^{(\mathrm{s})}=\widetilde{c}_{\Gamma} \frac{w^{-2 \epsilon}}{\epsilon^{2}} \mu^{2 \epsilon} s_{12}^{-1-\epsilon} \frac{\sin (2 \pi \epsilon)}{2 \pi \epsilon}, \\
& I_{1}^{(\mathrm{c})}=\widetilde{c}_{\Gamma} \frac{1-w^{-2 \epsilon}}{2 \epsilon^{2}} \mu^{2 \epsilon} s_{12}^{-1-\epsilon} \frac{\sin (2 \pi \epsilon)}{2 \pi \epsilon}, \\
& I_{2}^{(\mathrm{c})}=\widetilde{c}_{\Gamma} \frac{\mu^{2 \epsilon}}{4 \epsilon^{2}} s_{12}^{-1-\epsilon}\left(1+\frac{4^{\epsilon} \Gamma(1-2 \epsilon)}{\Gamma^{2}(1-\epsilon)}\right)\left[e^{i 2 \pi \epsilon}-w^{-2 \epsilon}{ }_{2} F_{1}\left(2 \epsilon, 2 \epsilon, 1+2 \epsilon ;-\frac{1}{w}\right) \frac{\sin (2 \pi \epsilon)}{2 \pi \epsilon}\right],
\end{aligned}
$$

with ${ }_{2} F_{1}(2 \epsilon, 2 \epsilon, 1+2 \epsilon ; z)=1+4 \epsilon^{2} \operatorname{Li}_{2}(z)+\mathcal{O}\left(\epsilon^{3}\right)$. As expected, the soft integral $I_{1}^{(\mathrm{s})}$ contains double poles in $\epsilon$, while the collinear integrals $I_{1}^{(\mathrm{c})}$ and $I_{2}^{(\mathrm{c})}$ develop single poles only. Each integral depends on the cut $w$, but the IR poles of the sum are independent of $w$ and they agree with the total divergences of the full integral in eq. (3.7).

At this stage, we have shown for the first time with a concrete example that the IR singularities in the LTD representation are constrained to a compact region of the loop three-momentum, as it was anticipated before based on the general demonstration from ref. [18], and we have recalculated the IR poles only by taking into account this compact region. The definition of the soft and collinear integrals is, however, somehow arbitrary; strictly, the IR singularities of the loop integrand are constrained to the region defined by the limit $w \rightarrow 0$ and more precisely to the segment defined by $\xi_{z} \in[-1,0]$. Any compact region of the loop three-momentum enclosing $\xi_{z} \in[-1,0]$ will lead to the same $\epsilon$ poles, although to different finite contributions. We will later redefine the IR regions of the loop integrand in a more convenient way to combine them with the real corrections.

Outside the soft and collinear regions, the sum of the dual integrals is finite because the integrand singularities appear at the intersection of forward light-cones. We define first a forward region with $v_{i} \leq 1 / 2$ :

$$
\begin{aligned}
I^{(\mathrm{f})}= & I_{1}\left(\xi_{1,0} \geq 1 ; v_{1} \leq \frac{1}{2}\right)+I_{2}\left(v_{2} \leq \frac{1}{2}\right)+I_{3}\left(v_{3} \leq \frac{1}{2}\right) \\
= & c_{\Gamma} \frac{1}{s_{12}} \int_{0}^{\infty} d \xi_{0} \int_{0}^{1 / 2} d v\left[\frac { 1 } { 1 + \xi _ { 0 } } \left((1-v)^{-1}\right.\right. \\
& \left.\left.+2 \log \left(\frac{1+\xi_{0}}{\xi_{0}}\right) \delta(v)\right)+\frac{(1-v)^{-1}}{1-\xi_{0}+i 0}\right]+\mathcal{O}(\epsilon) .
\end{aligned}
$$


The dual integrals $I_{1}$ and $I_{3}$ are divergent at $v_{1}=v_{3}=0$ in the forward region, but their sum is finite in this collinear limit. However, they diverge in the UV in all directions in spite of the absence of UV divergences in the original integral. This is due to the fact that dual propagators are linear in the loop momentum. The UV finiteness of the original integral is recovered by adding together all the dual integrals [15]. Therefore, to cancel this UV behaviour it is necessary to add the contribution of $I_{2}$. The integral over the sum of the three dual integrands in eq. (3.10) can thus be performed with $\epsilon=0$. In this expression, we have also identified all the angular and energy variables, $v_{i}=v, \xi_{1,0}-1=\xi_{2,0}=\xi_{3,0}=\xi_{0}$, to obtain common integration limits. Notice that it is necessary to keep the dual $i 0$ prescription of $I_{2}$ because its threshold singularity at $\xi_{2,0}=1$ appears within the integration region; it does not affect the UV cancellation. The logarithmic term of the integrand in eq. (3.10) is the result of the mismatch in the $\epsilon$-expansion of the integration measure of $I_{1}$ and $I_{3}$. The same result is obtained without extra logarithmic contributions by expressing $\left(\xi_{1,0}, v_{1}\right)$ in terms of $\left(\xi_{3,0}, v_{3}\right)$ at the price, however, of introducing cumbersome integration limits. We obtain in both cases

$$
I^{(\mathrm{f})}=c_{\Gamma} \frac{1}{s_{12}}\left[\frac{\pi^{2}}{3}-i \pi \log (2)\right]+\mathcal{O}(\epsilon) .
$$

The other finite contribution in the backward region $\left(v_{i} \geq 1 / 2\right)$ is

$$
\begin{aligned}
I^{(\mathrm{b})} & =I_{1}\left(\xi_{1,0} \geq w ; v_{1} \geq \frac{1}{2}\right)+I_{2}\left(\xi_{2,0} \geq 1+w ; v_{2} \geq \frac{1}{2}\right)+I_{3}\left(v_{3} \geq \frac{1}{2}\right) \\
& =c_{\Gamma} \frac{1}{s_{12}}\left[2 \operatorname{Li}_{2}\left(-\frac{1}{w}\right)-\log (2) \log (w)\right]+\mathcal{O}(\epsilon) .
\end{aligned}
$$

Now, the collinear cancellation occurs at $v_{1}=1=v_{2}$ and the UV behaviour of $I_{1}+I_{2}$ is cancelled by $I_{3}$. As expected, the sum of the soft, collinear, forward and backward integrals in eq. (3.9), eq. (3.11) and eq. (3.12) leads to the full result given in eq. (3.7), up to $\mathcal{O}(\epsilon)$.

\section{Real-Virtual mapping and cancellation of infrared singularities}

In the previous section we have analyzed the divergent structure of a scalar one-loop integral, and have isolated its IR behaviour to a compact region of the loop three-momentum. We shall illustrate now how to combine virtual corrections with real radiation in order to obtain physical cross-sections. To show the advantages of the method it is enough to work out a simplified calculation where the one-loop scattering amplitude is just given by the scalar three-point function

$$
\left|\mathcal{M}^{(1)}\left(p_{1}, p_{2} ; p_{3}\right)\right\rangle=-i g^{3} s_{12} L^{(1)}\left(p_{1}, p_{2},-p_{3}\right),
$$

where $g$ is an arbitrary coupling. Explicit computations in a real physical model will be presented elsewhere [29, 30]. The corresponding scalar tree-level amplitude is $\left|\mathcal{M}^{(0)}\left(p_{1}, p_{2} ; p_{3}\right)\right\rangle=i g$, and we shall take into account their interference, $\operatorname{Re}\left\langle\mathcal{M}^{(0)} \mid \mathcal{M}^{(1)}\right\rangle$, integrated over the two-body phase-space. We consider also scalar scattering amplitudes 
with emission of an extra particle $\left|\mathcal{M}_{i r}^{(0)}\left(p_{1}^{\prime}, p_{2}^{\prime}, p_{r}^{\prime} ; p_{3}\right)\right\rangle=-i g^{2} \sqrt{s_{12}} / s_{i r}^{\prime}$, and in particular the interference (see figure 1 (right))

$$
\operatorname{Re}\left\langle\mathcal{M}_{2 r}^{(0)} \mid \mathcal{M}_{1 r}^{(0)}\right\rangle=g^{4} \frac{s_{12}}{s_{1 r}^{\prime} s_{2 r}^{\prime}},
$$

which is integrated over the corresponding three-body phase-space. ${ }^{1}$ To combine virtual and real corrections directly, we first decompose the real-emission phase-space by using the identity $1=\theta\left(y_{2 r}^{\prime}-y_{1 r}^{\prime}\right)+\theta\left(y_{1 r}^{\prime}-y_{2 r}^{\prime}\right)$, where $y_{i r}^{\prime}=s_{i r}^{\prime} / s_{12}$. It is unnecessary to single out a soft region. In general, segmenting the real-emission phase-space into different regions featuring at most one soft and/or collinear singularity is a common practice in many subtraction schemes, as for instance in FKS [8]. The main novelty of the LTD approach is that that segmentation will be translated into the virtual contributions such that a local cancellation of singularities at the integrand level is achieved.

Thus, we define the following dual contributions to the total cross-section

$$
\begin{aligned}
\widetilde{\sigma}_{\mathrm{R}, i}^{(1)} & =\frac{1}{2 s_{12}} \int d \Phi_{1 \rightarrow 3} 2 \operatorname{Re}\left\langle\mathcal{M}_{2 r}^{(0)} \mid \mathcal{M}_{1 r}^{(0)}\right\rangle \theta\left(y_{j r}^{\prime}-y_{i r}^{\prime}\right), \\
\widetilde{\sigma}_{\mathrm{V}, i}^{(1)} & =\frac{1}{2 s_{12}} \int d \Phi_{1 \rightarrow 2} 2 \operatorname{Re}\left\langle\mathcal{M}^{(0)} \mid \mathcal{M}_{i}^{(1)}\right\rangle \theta\left(y_{j r}^{\prime}-y_{i r}^{\prime}\right), \quad i, j=1,2,
\end{aligned}
$$

where $\left|\mathcal{M}_{i}^{(1)}\right\rangle=-i g^{3} s_{12} I_{i}$ is the $i^{\text {th }}$ dual component of the one-loop scattering amplitude. The third dual component given by $I_{3}$ in eq. (3.3) does not contribute to the IR structure of $\left|\mathcal{M}^{(1)}\right\rangle$, and can be integrated independently of the real corrections.

The overall picture presented in eq. (4.3) must be completed with a mapping of the virtual and the real kinematics. For a fixed value of the external momenta $p_{1}$ and $p_{2}$ entering the loop scattering amplitude, and the loop three-momentum $\boldsymbol{\ell}$, we define the following mapping, with $q_{1}=\ell+p_{1}$ on-shell,

$$
\begin{array}{ll}
p_{r}^{\prime \mu}=q_{1}^{\mu}, & p_{1}^{\prime \mu}=-q_{3}^{\mu}+\alpha_{1} p_{2}^{\mu}=p_{1}^{\mu}-q_{1}^{\mu}+\alpha_{1} p_{2}^{\mu}, \\
p_{2}^{\prime \mu}=\left(1-\alpha_{1}\right) p_{2}^{\mu}, & \alpha_{1}=\frac{q_{3}^{2}}{2 q_{3} \cdot p_{2}},
\end{array}
$$

where $q_{3}=q_{1}-p_{1}$ is off-shell. Momentum conservation is automatically fulfilled as $p_{1}^{\prime}+$ $p_{2}^{\prime}+p_{r}^{\prime}=p_{1}+p_{2}$, and all the primed final-state momenta are on-shell. The non-collinear parton $p_{2}^{\prime}$ plays here the role of the spectator parton in the dipole formalism [6]. The mapping in eq. (4.4) is also valid for an arbitrary number of external particles, provided that $p_{i}^{\prime}=p_{i}$ for partons not entering in the collinear subprocess. The two-body invariants are given by

$$
y_{1 r}^{\prime}=\frac{v_{1} \xi_{1,0}}{1-\left(1-v_{1}\right) \xi_{1,0}}, \quad y_{2 r}^{\prime}=\frac{\left(1-v_{1}\right)\left(1-\xi_{1,0}\right) \xi_{1,0}}{1-\left(1-v_{1}\right) \xi_{1,0}}, \quad y_{12}^{\prime}=1-\xi_{1,0} .
$$

These expressions are obtained from the momentum parametrizations given in eq. (3.2) and eq. (4.4). The loop momentum $q_{1}$ and $p_{1}$ become collinear at $v_{1} \rightarrow 0$, with $\alpha_{1} \rightarrow 0$

\footnotetext{
${ }^{1}$ For identical final-state particles all the other possible interferences should also be considered. Besides, the squared amplitudes $\left|\mathcal{M}_{i r}\right|^{2}$ are necessary, which have to be mapped to self-energy insertions of external legs. For the current illustrative purposes of the paper, however, it is enough to consider this interference.
} 
and $y_{1 r}^{\prime} \rightarrow 0$ in this limit. According to eq. (4.3), we use this mapping in the regions of the two-body and three-body phase-space where $y_{1 r}^{\prime}<y_{2 r}^{\prime}$ :

$$
\begin{aligned}
\widetilde{\sigma}_{1}^{(1)}=\widetilde{\sigma}_{\mathrm{V}, 1}^{(1)}+\widetilde{\sigma}_{\mathrm{R}, 1}^{(1)}= & \sigma^{(0)} 2 g^{2} \int d\left[\xi_{1,0}\right] d\left[v_{1}\right] \theta\left(1-2 v_{1}\right) \theta\left(\frac{1-2 v_{1}}{1-v_{1}}-\xi_{1,0}\right) \\
& \times \xi_{1,0}^{-1}\left(v_{1}\left(1-v_{1}\right)\right)^{-1}\left[\left(\frac{1-\xi_{1,0}}{1-\left(1-v_{1}\right) \xi_{1,0}}\right)^{-2 \epsilon}-1\right],
\end{aligned}
$$

where $\sigma^{(0)}=g^{2} /\left(2 s_{12}\right) \int d \Phi_{1 \rightarrow 2}$ would be the lowest order cross-section. In eq. (4.6), the three-body phase-space $d \Phi_{1 \rightarrow 3}$ was written in terms of the loop variables $\xi_{1,0}$ and $v_{1}$ by using eq. (4.5). The integrand in eq. (4.6) has the form $\xi_{1,0}^{-1-2 \epsilon} v_{1}^{-1-\epsilon} f\left(v_{1}, \xi_{1,0}\right)$, but $f\left(v_{1}=0\right)=0=f\left(\xi_{1,0}=0\right)$ with $f\left(v_{1}, \xi_{1,0}\right)=\mathcal{O}(\epsilon)$, and thus this integral is of order $\epsilon$, i.e. $\widetilde{\sigma}_{1}^{(1)}=\mathcal{O}(\epsilon)$. Similarly, we define a complementary mapping to cover the remaining three-body phase-space, which includes the other collinear limit with $y_{2 r}^{\prime} \rightarrow 0$. Considering $q_{2}$ on-shell, the corresponding mapping is

$$
\begin{array}{rlrl}
p_{2}^{\prime \mu} & =q_{2}^{\mu}, & p_{r}^{\prime \mu} & =-q_{1}^{\mu}+\alpha_{2} p_{1}^{\mu}=p_{2}^{\mu}-q_{2}^{\mu}+\alpha_{2} p_{1}^{\mu}, \\
p_{1}^{\prime \mu}=\left(1-\alpha_{2}\right) p_{1}^{\mu}, & \alpha_{2}=\frac{q_{1}^{2}}{2 q_{1} \cdot p_{1}},
\end{array}
$$

and the associated invariants are

$$
y_{12}^{\prime}=\frac{v_{2}\left(1-\xi_{2,0}\right) \xi_{2,0}}{1-v_{2} \xi_{2,0}}, \quad y_{2 r}^{\prime}=\frac{\left(1-v_{2}\right) \xi_{2,0}}{1-v_{2} \xi_{2,0}}, \quad y_{1 r}^{\prime}=1-\xi_{2,0} .
$$

Then,

$$
\begin{aligned}
\widetilde{\sigma}_{2}^{(1)}=\widetilde{\sigma}_{\mathrm{V}, 2}^{(1)}+\widetilde{\sigma}_{\mathrm{R}, 2}^{(1)}= & \sigma^{(0)} 2 g^{2} \int d\left[\xi_{2,0}\right] d\left[v_{2}\right] \theta\left(\frac{1-\sqrt{1-v_{2}}}{v_{2}}-\xi_{2,0}\right)\left(1-v_{2}\right)^{-1} \\
& \times\left[\frac{\left(1-\xi_{2,0}\right)^{-2 \epsilon}}{\left(1-v_{2} \xi_{2,0}\right)^{1-2 \epsilon}}-\frac{1}{1-\xi_{2,0}+i 0}-i \pi \delta\left(1-\xi_{2,0}\right)\right],
\end{aligned}
$$

and we obtain directly with $\epsilon=0$

$$
\widetilde{\sigma}_{2}^{(1)}=-\sigma^{(0)} a \frac{\pi^{2}}{6}+\mathcal{O}(\epsilon)
$$

where $a=g^{2} /(4 \pi)^{2}$. The condition $y_{2 r}^{\prime}<y_{1 r}^{\prime}$ excludes the threshold singularity of $I_{2}$ from the integration region with the exception of the single point at $v_{2}=1, \xi_{2.0}=1$. This makes unnecessary the introduction of an energy-cut $w$ as in section 3 .

Finally, analogously to the forward and backward dual integrals defined in section 3, we collect the remnants of the three dual integrals obtained after excluding the respective regions of the loop three-momentum already included in eq. (4.6) and eq. (4.10). Explicitly, we define

$$
\begin{aligned}
\bar{\sigma}_{\mathrm{V}}^{(1)}= & \sigma^{(0)} 2 g^{2}\left[-\int d\left[\xi_{1,0}\right] d\left[v_{1}\right]\left(1-\theta\left(1-2 v_{1}\right) \theta\left(\frac{1-2 v_{1}}{1-v_{1}}-\xi_{1,0}\right)\right) \xi_{1,0}^{-1}\left(v_{1}\left(1-v_{1}\right)\right)^{-1}\right. \\
& -\int d\left[\xi_{2,0}\right] d\left[v_{2}\right] \theta\left(\xi_{2,0}-\frac{1-\sqrt{1-v_{2}}}{v_{2}}\right)\left(1-v_{2}\right)^{-1}\left(\frac{1}{1-\xi_{2,0}+i 0}+i \pi \delta\left(1-\xi_{2,0}\right)\right) \\
& \left.+\int d\left[\xi_{3,0}\right] d\left[v_{3}\right] \frac{v_{3}^{-1}}{1+\xi_{3,0}}\right] .
\end{aligned}
$$


Although the individual contributions in eq. (4.11) are singular, this virtual cross-section is UV and IR finite, and can be calculated with $\epsilon=0$. We apply the change of variables used to obtain eq. (3.11) and eq. (3.12) to avoid logarithmic contributions from the $\epsilon$-expansion of the integration measure. The result is

$$
\bar{\sigma}_{\mathrm{V}}^{(1)}=\sigma^{(0)} a \frac{\pi^{2}}{6}+\mathcal{O}(\epsilon) .
$$

The sum of all the contributions, eq. (4.6), eq. (4.10) and eq. (4.12), gives a total crosssection of $\mathcal{O}(\epsilon)$, in agreement with the result that would be obtained from the standard calculation in DREG.

To conclude this section, we want to emphasize that DREG was only used for a consistency check of our results. The advantage of the method presented here is that NLO cross-sections can be computed in four dimensions, i.e. with $\epsilon=0$, because soft and collinear divergences of virtual and real corrections are matched locally at the integrand level. Collinear factorization guarantees that this matching is fulfilled in QCD [18, 27]. Threshold singularities should be treated apart in numerical calculations, e.g. by contour deformation $[23,28]$. A first numerical implementation of multi-leg scalar and tensor oneloop integrals in LTD has been presented in refs. [19-21].

\section{Ultraviolet renormalization}

LTD also offers an appealing physical interpretation for the renormalization of UV divergences at the integrand level. To illustrate the cancellation of these divergences, we consider the simplest purely UV scalar two-point function, with massless internal lines. A detailed calculation of this scalar two-point function in the LTD framework has been presented in ref. [15]. Following the suggestion of ref. [28], we construct its UV counter-term from

$$
I_{\mathrm{UV}}^{\mathrm{cnt}}=\int_{\ell} \frac{1}{\left(q_{\mathrm{UV}}^{2}-\mu_{\mathrm{UV}}^{2}+i 0\right)^{2}},
$$

where $q_{\mathrm{UV}}=\ell+k_{\mathrm{UV}}$, with $k_{\mathrm{UV}}$ located somewhere in the loop momentum space. This counter-term has the same local UV behaviour as the integrand of the scalar two-point function in all the loop directions. We shall now find the dual representation of $I_{\mathrm{UV}}^{\text {cnt }}$ in three dimensions.

The dual representation of eq. (5.1) requires to deal with double poles in the loop energy [17], with the UV propagator being massive. The calculation of the corresponding residue leads to the dual representation

$$
I_{\mathrm{UV}}^{\mathrm{cnt}}=\int_{\ell} \frac{\tilde{\delta}\left(q_{\mathrm{UV}}\right)}{2\left(q_{\mathrm{UV}, 0}^{(+)}\right)^{2}},
$$

where $q_{\mathrm{UV}, 0}^{(+)}=\sqrt{\mathbf{q}_{\mathrm{UV}}^{2}+\mu_{\mathrm{UV}}^{2}-i 0}$. The detailed calculation of the double pole residue has been presented in ref. [17]; again we used $\eta^{\mu}=(1,0)$ as dual vector. The arbitrary scale $\mu_{\mathrm{UV}}$ 


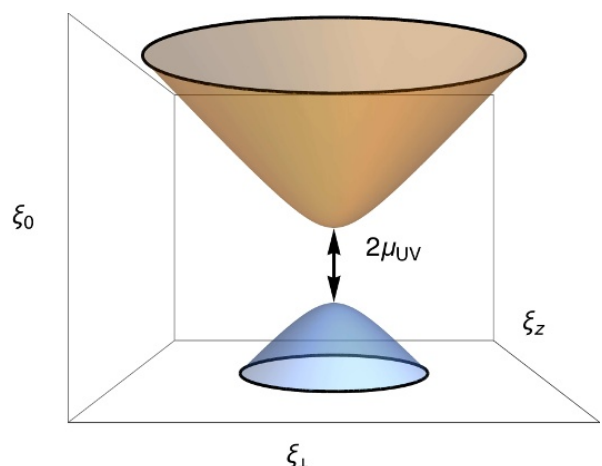

Figure 3. On-shell hyperboloids of the ultraviolet counter-term.

admits a direct physical interpretation as renormalization scale because the UV counterterm only contributes for loop energies larger than $-k_{\mathrm{UV}, 0}+\mu_{\mathrm{UV}}$ in the LTD representation (see figure 3), although with unconstrained loop three-momentum. As renormalization scale, $\mu_{\mathrm{UV}}$ has to be chosen of the order of the physical hard scale. In addition, a clear criteria to select $\mu_{\mathrm{UV}}$ and $k_{\mathrm{UV}}$ arises if we pretend that the on-shell hyperboloids of the UV propagator in eq. (5.1) do not intersect with any of the forward on-shell hyperboloids of the original integral. Since the distance between the UV forward and backward on-shell hyperboloids is $2 \mu_{\mathrm{UV}}$, the minimal choice fulfilling the aforementioned conditions would be $1 / 2$ of the hard scale, which accidentally agrees with one of the standard limits used to estimate the theoretical uncertainty. Of course, this is an ad hoc argument that is not supported by any physical evidence, but it represents a good interpretation of that choice.

In consequence, subtracting the UV counter-term from the scalar two-point function we obtain

$$
L^{(1)}(p,-p)-I_{\mathrm{UV}}^{\mathrm{cnt}}=\frac{1}{(4 \pi)^{2}}\left[-\log \left(-\frac{p^{2}}{\mu_{\mathrm{UV}}^{2}}-i 0\right)+2\right]+\mathcal{O}(\epsilon)
$$

and this calculation gives the same result if each of the individual contributions is integrated in DREG or if the sum of the integrands is taken at $\epsilon=0$.

We conclude this section by noting that contrary to the four-dimensional UV renormalization introduced in ref. [24], where the limit $\mu \rightarrow 0$ is taken with the purpose of removing the polynomial dependence in $\mu$ and the remainder is evaluated at the renormalization scale $\mu=\mu_{\mathrm{R}}$, the counter-term defined by eq. (5.1) keeps the complete dependence in $\mu_{\mathrm{UV}}$. Eventual polynomial dependences in $\mu_{\mathrm{UV}}$ can be cancelled by adding to the counter-term UV subleading contributions such that only the UV pole is subtracted [28].

\section{Conclusions}

We have applied the LTD formalism to physically reinterpret perturbative calculations in QFT. With some reference examples, we have reanalyzed the infrared and causal structure of NLO computations. The sum over degenerate IR states is accomplished at integrand level by mapping the phase-space of real radiation with that of the virtual contributions 
in the region of the loop three-momentum which is of the size of the physical scales. This represents a new paradigm in perturbative calculations as virtual and real corrections are not regularized independently, but they act directly as the IR subtraction counter-term of each other. Outside this region, at large loop three-momentum, virtual corrections require only the subtraction of UV divergences at integrand level to render them finite and renormalization acquires a clear physical interpretation.

The application of LTD to loop scattering amplitudes in gauge theories was already discussed in ref. [15], and the momentum mappings introduced in this paper can be extended straightforwardly to massless $n$-body scattering processes, and even generalized to the massive case. Each mapping is suitable for a different collinear configuration, and has to be applied to the sum of diagrams belonging to gauge invariant subsets featuring the same collinear limit.

The results presented in this paper represent a promising first effort towards a fourdimensional implementation for the computation of physical cross-sections at NLO without introducing soft and final-state collinear subtractions. They also open the attractive possibility of extending this approach to next-to-next-to leading order (NNLO) and beyond. Further details will be given in a forthcoming publication, including the application to gauge theories [29].

\section{Acknowledgments}

We thank S. Catani for a longstanding fruitful collaboration. This work has been supported by the Research Executive Agency (REA) under the Grant Agreement No. PITNGA-2010-264564 (LHCPhenoNet), by CONICET Argentina, by the Spanish Government and ERDF funds from the European Commission (Grants No. FPA2014-53631-C2-1-P, FPA2011-23778, CSD2007-00042 Consolider Project CPAN) and by Generalitat Valenciana under Grant No. PROMETEOII/2013/007. The work of RJHP is partially supported by CONACyT, México.

Open Access. This article is distributed under the terms of the Creative Commons Attribution License (CC-BY 4.0), which permits any use, distribution and reproduction in any medium, provided the original author(s) and source are credited.

\section{References}

[1] C.-N. Yang and R.L. Mills, Conservation of Isotopic Spin and Isotopic Gauge Invariance, Phys. Rev. 96 (1954) 191 [INSPIRE].

[2] C.G. Bollini and J.J. Giambiagi, Dimensional Renormalization: The Number of Dimensions as a Regularizing Parameter, Nuovo Cim. B 12 (1972) 20 [INSPIRE].

[3] G. 't Hooft and M.J.G. Veltman, Regularization and Renormalization of Gauge Fields, Nucl. Phys. B 44 (1972) 189 [INSPIRE].

[4] G.M. Cicuta and E. Montaldi, Analytic renormalization via continuous space dimension, Lett. Nuovo Cim. 4 (1972) 329 [INSPIRE]. 
[5] J.F. Ashmore, A Method of Gauge Invariant Regularization, Lett. Nuovo Cim. 4 (1972) 289 [INSPIRE].

[6] S. Catani and M.H. Seymour, A General algorithm for calculating jet cross-sections in NLO QCD, Nucl. Phys. B 485 (1997) 291 [Erratum ibid. B 510 (1998) 503] [hep-ph/9605323] [INSPIRE].

[7] S. Catani and M.H. Seymour, The dipole formalism for the calculation of QCD jet cross-sections at next-to-leading order, Phys. Lett. B 378 (1996) 287 [hep-ph/9602277] [INSPIRE].

[8] S. Frixione, Z. Kunszt and A. Signer, Three jet cross-sections to next-to-leading order, Nucl. Phys. B 467 (1996) 399 [hep-ph/9512328] [INSPIRE].

[9] A. Gehrmann-De Ridder, T. Gehrmann and E.W.N. Glover, Antenna subtraction at NNLO, JHEP 09 (2005) 056 [hep-ph/0505111] [INSPIRE].

[10] S. Catani and M. Grazzini, An NNLO subtraction formalism in hadron collisions and its application to Higgs boson production at the LHC, Phys. Rev. Lett. 98 (2007) 222002 [hep-ph/0703012] [INSPIRE].

[11] V. Del Duca, C. Duhr, G. Somogyi, F. Tramontano and Z. Trócsányi, Higgs boson decay into b-quarks at NNLO accuracy, JHEP 04 (2015) 036 [arXiv:1501.07226] [INSPIRE].

[12] M. Czakon, A novel subtraction scheme for double-real radiation at NNLO, Phys. Lett. B 693 (2010) 259 [arXiv: 1005.0274] [INSPIRE].

[13] R. Boughezal, C. Focke, X. Liu and F. Petriello, $W$-boson production in association with a jet at next-to-next-to-leading order in perturbative QCD, Phys. Rev. Lett. 115 (2015) 062002 [arXiv: 1504.02131] [INSPIRE].

[14] J. Gaunt, M. Stahlhofen, F.J. Tackmann and J.R. Walsh, N-jettiness Subtractions for NNLO QCD Calculations, JHEP 09 (2015) 058 [arXiv: 1505.04794] [INSPIRE].

[15] S. Catani, T. Gleisberg, F. Krauss, G. Rodrigo and J.-C. Winter, From loops to trees by-passing Feynman's theorem, JHEP 09 (2008) 065 [arXiv: 0804.3170] [INSPIRE].

[16] I. Bierenbaum, S. Catani, P. Draggiotis and G. Rodrigo, A Tree-Loop Duality Relation at Two Loops and Beyond, JHEP 10 (2010) 073 [arXiv: 1007.0194] [InSPIRE].

[17] I. Bierenbaum, S. Buchta, P. Draggiotis, I. Malamos and G. Rodrigo, Tree-Loop Duality Relation beyond simple poles, JHEP 03 (2013) 025 [arXiv: 1211.5048] [INSPIRE].

[18] S. Buchta, G. Chachamis, P. Draggiotis, I. Malamos and G. Rodrigo, On the singular behaviour of scattering amplitudes in quantum field theory, JHEP 11 (2014) 014 [arXiv: 1405.7850] [INSPIRE].

[19] S. Buchta, Theoretical foundations and applications of the Loop-Tree Duality in Quantum Field Theories, Ph.D. Thesis, Universitat de València, (2015), arXiv:1509.07167 [INSPIRE].

[20] S. Buchta, G. Chachamis, P. Draggiotis, I. Malamos and G. Rodrigo, Towards a Numerical Implementation of the Loop-Tree Duality Method, Nucl. Part. Phys. Proc. 258-259 (2015) 33 [arXiv:1509.07386] [INSPIRE].

[21] S. Buchta, G. Chachamis, P. Draggiotis and G. Rodrigo, Numerical implementation of the Loop-Tree Duality method, arXiv:1510.00187 [INSPIRE].

[22] G.F.R. Sborlini, R. Hernandez-Pinto and G. Rodrigo, From dimensional regularization to NLO computations in four dimensions, PoS (EPS-HEP2015) 479 [arXiv: 1510.01079] [INSPIRE]. 
[23] D.E. Soper, QCD calculations by numerical integration, Phys. Rev. Lett. 81 (1998) 2638 [hep-ph/9804454] [INSPIRE].

[24] R. Pittau, A four-dimensional approach to quantum field theories, JHEP 11 (2012) 151 [arXiv: 1208.5457] [INSPIRE].

[25] A.M. Donati and R. Pittau, Gauge invariance at work in FDR: $H \rightarrow \gamma \gamma$, JHEP 04 (2013) 167 [arXiv: 1302.5668] [INSPIRE].

[26] R.A. Fazio, P. Mastrolia, E. Mirabella and W.J. Torres Bobadilla, On the Four-Dimensional Formulation of Dimensionally Regulated Amplitudes, Eur. Phys. J. C 74 (2014) 3197 [arXiv: 1404.4783] [INSPIRE].

[27] S. Catani, D. de Florian and G. Rodrigo, Space-like (versus time-like) collinear limits in QCD: Is factorization violated?, JHEP 07 (2012) 026 [arXiv: 1112.4405] [INSPIRE].

[28] S. Becker, C. Reuschle and S. Weinzierl, Numerical NLO QCD calculations, JHEP 12 (2010) 013 [arXiv: 1010.4187] [INSPIRE].

[29] G. Rodrigo et al., IFIC/15-73 in preparation.

[30] G.F.R. Sborlini, Loop-tree duality and quantum field theory in four dimensions, PoS (RADCOR2015) 082 [arXiv: 1601.04634] [INSPIRE]. 